Recepción: 10/ 08 / 2018

Aceptación: 22 / 10 / 2018

Publicación: 03 / 12 / 2018
Ciencias de la salud

Artículo científico

\title{
Política de salud ambiental, efectos en la salud humana. Guayaquil. Ecuador
}

\author{
Environmental health policy, effects on human health. Guayaquil. Ecuador
}

\section{Política de saúde ambiental, efeitos na saúde humana. Guayaquil equador}

\author{
Delia N. Crespo-Antepara ${ }^{\text {I }}$ \\ deliacrespo57@hotmail.com
}

Guillermina L. Gómez Guanga II guillita_gomez@hotmail.com

\section{Correspondencia: deliacrespo57@hotmail.com}

I Magíster en Gerencia Clínica en Salud Sexual y Reproductiva, Obstetriz, Docente de la Universidad de Guayaquil, Guayaquil, Ecuador.

II Licenciada en Nutrición Dietética y Estética, Docente de la Universidad de Guayaquil, Guayaquil, Ecuador. 


\section{Resumen}

La salud ambiental comprende aspectos de la salud humana, en donde las dimensiones de la calidad de vida y el bienestar social son requisitos indispensables en los constructos narrativos y praxiológicos, la salud ambiental es determinada por factores físicos; químicos, biológicos, sociales y psico-sociales. Se realizó una revisión bibliográfica de la literatura existente relacionada con el tema, con la finalidad de interpretar la política nacional de salud ambiental en el Ecuador, desde su evolución histórica y contextual, describiendo la situación de la salud en la región y contrastándolas entre sí, identificando los factores de riesgo en la ciudad de Guayaquil y los efectos en la salud humana que permitan generar una alternativa sustentable y sostenible para la Pacha Mama. La investigación busca encontrar los intersticios desde donde se observe, analice y critique las políticas de salud ambiental en el Ecuador, específicamente en la ciudad de Guayaquil, se realiza un recorrido histórico desde la década de los 70 hasta la fecha, se compara con los objetivos de las políticas emitidas por otros países de la región y se ancla en la actual ley emitida por los organismos gubernamentales, Se presenta una propuesta humanista desde la Descolonización de la docencia universitaria como punta de lanza que genere la toma de conciencia y de construcción de la sociedad en personas humana desde el Sur hacia el planeta.

Palabras clave: salud ambiental; calidad de vida; bienestar social; política gubernamental; sumak kawsay; humanismo; decolonización de la docencia universitaria.

\section{Abstract}

Environmental health includes aspects of human health, where the dimensions of quality of life and social welfare are essential requirements in narrative and praxiological constructs, environmental health is determined by physical factors; chemical, biological, social and psychosocial. A literature review of the existing literature related to the subject was carried out, with the purpose of interpreting the national environmental health policy in Ecuador, from its historical and contextual evolution, describing the health situation in the region and contrasting them with each other, identifying the risk factors in the city of Guayaquil and the effects on human health that allow generating a sustainable and sustainable alternative for the Pacha Mama. The research seeks to find the interstices from where environmental health policies are observed, analyzed and criticized in Ecuador, specifically in the city of Guayaquil, a historical journey is made from the 
70 's to date, compared with the objectives of the policies issued by other countries in the region and is anchored in the current law issued by government agencies. A humanist proposal is presented from the decolonization of university teaching as a spearhead that generates awareness and construction of the society in human persons from the South to the planet.

Key words: environmental health; quality of life; social welfare; government policy; Sumak kawsay; humanism; decolonization of university teaching.

\section{Resumo}

A saúde ambiental inclui aspectos da saúde humana, onde as dimensões de qualidade de vida e bem-estar social são requisitos essenciais nos construtos narrativos e praxiológicos, a saúde ambiental é determinada por fatores físicos; química, biológica, social e psicossocial. Uma revisão da literatura de literatura relacionada foi realizada com o tema existente, a fim de interpretar a política nacional de saúde ambiental no Equador, desde a sua evolução histórica e contextual, descrevendo a situação da saúde na região e compará-los uns com os outros, identificar os fatores de risco na cidade de Guayaquil e os efeitos na saúde humana que permitem gerar uma alternativa sustentável e sustentável para a Pacha Mama. A pesquisa tem como objetivo encontrar as lacunas de onde você observar, analisar e criticar as políticas de saúde ambiental no Equador, especificamente na cidade de Guayaquil, um passeio histórico é a partir dos anos 70, até à data, em comparação com os objectivos apólices emitidas por outros países da região e é ancorados na legislação vigente emitida por agências governamentais, uma proposta humanista é apresentado a partir de Descolonização do ensino universitário como ponta de lança para gerar consciência e construção sociedade em pessoas humanas do Sul para o planeta.

Palavras chave: saúde ambiental; qualidade de vida; bem estar social; política governamental; sumak kawsay; humanismo descolonização do ensino universitário.

\section{Introducción}

La salud ambiental está relacionada con la salud humana, busca la relación intrínseca entre el hombre y la naturaleza, presenta una cosmovisión multidimensional debido a que encierra una serie de factores que permiten prever que, sin salud ambiental, el hombre está destinado a perecer, en cambio, sin salud humana, la salud ambiental estará mucho mejor. Es el hombre quien 
ha diseñado políticas para brindarle salud al ambiente, cuando es el quién con su codicia desmedida e irresponsabilidad incontrolada, intoxica día a día a la madre naturaleza1.

La investigación acomete una secuencia pertinente, a través de una evolución histórica del objeto de estudio, nos embarcamos en una travesía que toma su punto de partida en la década de los setenta en el siglo pasado de la historia ecuatoriana, desde allí se analizan las formas irracionales de explotación petrolera y minerales con que se obtuvieron riqueza sin tomar ninguna precaución en cuanto al maltrato de la naturaleza, se sigue el hilo de la historia y se recorre las diferentes constituciones en su parte pertinente de salud ambiental, se realiza una mirada al Ecuador de la post dictadura y a los artículos de la constitución que con un color pálido y demagógico enuncia en un artículo que la atención a la salud de la población y saneamiento ambiental serían manejados desde los organismos respectivos. Se enuncia el boom bananero como uno de los medios de producción más irresponsables en cuanto a salud ambiental de la región litoral.

En este recorrido retrospectivo, nos detenemos en la década de los ochenta y visualizamos el fenómeno de las migraciones internas de la zona rural a las grandes ciudades y la construcción de los anillos periféricos, que viene acompañados de miseria por la falta de empleo y falta de atención de los gobiernos seccionales en los servicios básicos lo cual genera deterioro en la salud ambiental de estas zonas, se observa la presión de los organismos internacionales y de ONG ambientalistas en el país y el débil intento del gobierno de generar normas de eco-desarrollo, se observa que ya en la década de los noventa se instala la defensa del patrimonio natural del país y se invita a la protección del medio ambiente con la planificación de programas de desarrollo sustentables y que irían en pertinencia con el desarrollo económico pero con visión humanista, se generan políticas de gestión de los recursos naturales mediante el uso de la tecnología, se permite la participación de estratos sociales antes excluidos, se empiezan a generar programas educativos en relación a la salud ambiental. Para este siglo, se aborda con absoluta responsabilidad desde la nueva y última constitución, la relación del ser humano con la Pacha Mama y se inscribe el nombre del Sumak Kawsay para ser tomado como paradigma y principio filosófico por los ecuatorianos, se crean políticas y leyes que trabajen bis a bis y sean congruentes con la constitución de Monte Cristi del 2008, sin embargo, nos daremos cuenta de la gran falacia política ya que todo es papel escrito y muerto, es retórica con tintes de patriotismo planetario y la 
salud ambiental es ultrajada al igual que los hombres y mujeres de los asentamientos indígenas del oriente ecuatoriano2.

Desde el contexto regional se comparan los objetivos de las políticas ambientales de países de Latinoamérica y nos posicionamos en el nicho guayaquileño, lugar de nuestro desempeño profesional, desde allí, se observa las políticas de salud ambiental que maneja el cabildo porteño con lo que se pretende promover y asegurar la gestión ambiental, sin embargo, por nuestra experiencia en el campo de la vinculación universitaria con la sociedad, podemos emitir juicio de valor al contrastar la realidad in situ con la realidad políticamente maquillada que nos presentan y hacen conocer.

Se discute la realidad con las políticas emitidas y se concuerda que existen muchas deficiencias en cuanto al tratamiento serio y responsable de la salud ambiental en el país y la ciudad de Guayaquil, por ello se emite una alternativa desde la Decolonización del docente universitario ecuatoriano, para que sea este, quién logre desarraigar la colonialidad impregnada en el constructo socio cognitivos de la población, hay que transformar la universidad para transformar la sociedad.

\section{Método}

Se realizó una revisión bibliográfica de la literatura existente relacionada con el tema, con la finalidad de interpretar la política nacional de salud ambiental en el Ecuador, desde su evolución histórica y contextual, describiendo la situación de la salud en la región y contrastándolas entre sí, identificando los factores de riesgo en la ciudad de Guayaquil y los efectos en la salud humana que permitan generar una alternativa sustentable y sostenible para la Pacha Mama.

\section{Desarrollo}

Los problemas ecológicos o ambientales en el plano histórico aparecieron con el capitalismo como sistema social, pues antes de esta formación socioeconómica, aunque el medio ambiente sufrió una transformación notable, tanto por la presencia de fenómenos naturales como por la actividad humana, los cambios realizados no rebasaron los límites o capacidades del ecosistema natural a escala planetaria, que determinaran su irreversibilidad. El capitalismo, al requerir cada 
vez en mayor medida la obtención de ganancias, convirtió el medio natural y sus recursos en fuente directa de objeto de explotación y consumo, igual que cualquier otra mercancía.

La relación que existe entre el estado de salud y el ambiente, incluye numerosos y complejos aspectos entre los cuales se consideran las exigencias básicas relacionadas con la salud ambiental, como es el caso del mantenimiento de las fuentes de agua, la cantidad y la calidad del abasto, la disposición adecuada de los residuales líquidos y sólidos, los alimentos inocuos y nutritivos, la vivienda segura, entre otros. El ambiente, valorado según el enfoque de ecosistema, incluyó los riesgos físicos, las posibles contaminaciones o exposiciones a agentes biológicos y químicos, pero también al componente psicosocial, al cual se asoció decisivamente la salud individual y colectiva.

\section{Evolución histórica}

Las políticas emitidas en relación a salud ambiental en el Ecuador, han sufrido algunos cambios, en la reconstrucción histórica que se realiza, desde la dictadura militar de la década de los setenta en el siglo próximo anterior, se puede evidenciar la inexistencia de políticas sobre salud ambiental salvo contados conatos de cuidar la salud de los habitantes a través de programas llenos de vacíos científico, siendo más concretos, en los años setenta no existieron políticas encaminadas a la preservación de la salud ambiental, al contrario, el pensamiento de los gobernantes de aquella época y el desconocimiento de la población en materia de salud ambiental, eran antagónicas a la ideología y filosofía de vida de los ecuatorianos en el presente. En la etapa de la dictadura militar desde los años 1972 de Rodríguez Lara, el triunvirato militar desde 1976 hasta la reconstitución de la democracia en 1979, se abusó indiscriminadamente de los recursos naturales y no se tomaron medidas para precautelar la salud de la Pacha Mama, sino que, se explotó sin el más mínimo cuidado de estos recursos.

En la década de los setenta en el siglo próximo anterior, los militares que gobernaron al país, cumplieron el rol de intervencionistas y reguladores de la función económica, manteniendo centralizada la gestión, planificación y aplicación de normativas, reglamentos, leyes y constitución que pudieron cumplir con el encargo social que deberían haber tenido. No existió una política de carácter holístico e integrador que sea congruente con la obtención de riqueza mediante la explotación de recursos naturales con la salud del medio ambiente en conjunto y por 
consecuencia la salud de la población ecuatoriana, principalmente de aquella población que vivía cerca de las minas o pozos de explotación petrolera. Fue una época de incertidumbre política ya que Velasco Ibarra, gobernó hasta el año 72 entre democracia y dictadura hasta el año 1978. El país se vio inmerso en una espiral capitalista, en donde el boom petrolero constituyó el eje del desarrollo económico, pero sin el manejo de la salud ambiental como compensación a la explotación de los recursos naturales.

En la constitución de 1978, con sus 144 artículos, no emite criterio específico sobre la salud ambiental, tan solo en el artículo 29 literal 2., enuncia:

La atención a la salud de la población y el saneamiento ambiental de las ciudades y el campo, por medio de la socialización de la medicina, de los diferentes organismos encargados de su ejecución y de la creación de la correspondiente infraestructura, de acuerdo con la ley; la aplicación de programas tendientes a eliminar el alcoholismo y otras toxicomanías y a disminuir la mortalidad infantil (4, p. 10).

Con la llegada de la democracia al Ecuador, se tenía la esperanza que la explotación petrolera y otros recursos minerales, sean racionalizados, que la producción bananera que estaba en apogeo, sea manejada con sostenibilidad y políticas de salud ambiental, sin embargo, nunca fue de esa forma. En la región oriental del Ecuador, se presentaron y hasta hoy en día existen secuelas en la salud ambiental de la región y por ende en la salud humana de sus habitantes, en lo que concierne a la zona litoral que es donde se produce el banano, la mala práctica de fumigación vía aérea, ocasionó muchas enfermedades en los pobladores y la contaminación del ambiente, situación que persiste hasta nuestros días. No se generó el llamado saneamiento ambiental que promueve el literal 2 del artículo 296.

En la década de los ochenta, el País contaba ya con 8`279.000 habitantes, la población empieza a desplazarse de la zona rural agrícola, a las grandes ciudades y con ello las consecuencias de los anillos periféricos principalmente en Guayaquil y Quito. Se suma a la falta de políticas en salud ambiental el problema de las migraciones internas que ocasionan congestión en cuanto a la prestación de servicios básicos como son agua potable, alcantarillado y disposición de excretas, esto ocurre fundamentalmente en Guayaquil que pertenece a la ciudad de Guayaquil, los hacinamientos humanos generan descontrol en la municipalidad de aquella época y la salud 
ambiental se ve afectada con este fenómeno migratorio. A pesar de este escenario, es preciso señalar que, debido a la presión de organismos internacionales, ONG ambientalistas del Ecuador y el Mundo, el gobierno asumió una política con claros síntomas de integrar la dimensión ambiental a las estrategias de desarrollo del país, se crearon normativas para el denominado ecodesarrollo, esto se pudo dar.

En la década de los noventa, se reconstruye la constitución y en su artículo 3. Se promulga "Defender el patrimonio natural y cultural del país y proteger el medio ambiente”, así, se enuncia la voluntad de otorgar a la dimensión ambiental un valor que antes no tenía, en estos años, se empieza a planificar en función de preservar el medio ambiente mediante la estructuración de planes de desarrollo, se incorpora al crecimiento económico, la visión de desarrollo humano y la conservación del patrimonio natural. En materia de salud ambiental se generan políticas de gestión de los recursos naturales mediante el uso de la tecnología, se permite la participación de estratos sociales antes excluidos, se empiezan a generar programas educativos en relación a la salud ambiental y se promueve la investigación para establecer modelos de consumo y explotación poco invasiva, además, se crean patrones de producción más limpios 8.

En la primera década de este siglo, es a partir del 2008, fecha en que se crea una nueva constitución, se promulgan sendos artículos en la carta magna que hace ilusionar al mundo entero en cuanto a la relación del ser humano con el planeta, es la constitución del Sumak Kawsay, en donde se da prioridad al tratamiento de la Pacha Mama de parte de los ecuatorianos, así en sus artículos 12, 13, 14 y 15 se habla de los derechos del buen vivir de los ciudadanos y de vivir en un ambiente sano, así, concretamente enuncia el artículo 14:

"Se reconoce el derecho de la población a vivir en un ambiente sano y ecológicamente equilibrado, que garantice la sostenibilidad y el buen vivir, sumak kawsay. Se declara de interés público la preservación del ambiente, la conservación de los ecosistemas, la biodiversidad y la integridad del patrimonio genético del país, la prevención del daño ambiental y la recuperación de los espacios naturales degradados". (8. p, 24).

Es en este artículo cuando se reconoce a la salud ambiental, la misma que como se indicó en el primer párrafo de la introducción tiene relación con la salud humana, con la calidad de vida y el bienestar social de los ciudadanos, es por esta razón que he considerado pertinente hacer alusión 
a la constitución ecuatoriana, con sus tantas modificaciones, ya que así, se puede entrar en el contexto de la salud ambiental, la misma que está determinada por factores ambientales de carácter físicos, químicos, biológicos y sociales. Al mencionar el artículo 14 que es derecho de los ecuatorianos vivir en un ambiente sano y ecológicamente equilibrado, es una concepción multidimensional, pues un ambiente sano es estar libre de contaminación, es invitar a los organismos gubernamentales y ciudadanos ecuatorianos a establecer pautas de comportamiento humanistas para con el planeta, es restringir la explotación irracional de los recursos naturales, pues con ello se genera contaminación del aire, de los ríos, se devasta la flora y se hace emigrar la fauna de su ecosistema, es amar la Pacha Mama y ser seres universalmente comprometidos con el planeta y sus seres vivos, es entrar en el paradigma del Sumak Kawsay de nuestros ancestros, es por ello que más adelante se hablará de la Decolonización del docente universitario.

El Artículo 15, es congruente con cada una de las partes de la constitución, es un aderezo al artículo 14, las especificaciones de lo que hacer y no hacer en materia de salud ambiental, es el llamado de atención a los gobernantes del estado y a los funcionarios públicos o privados, al uso racional de las tecnologías, es la invitación al uso de energías alternas no contaminantes, brinda a los ecuatorianos la seguridad de una soberanía alimentaria y el derecho irrenunciable al agua, así también, por otro lado, prohíbe toda actividad que afecte a la salud humana.

El artículo 31 resalta la importancia del disfrute pleno de la ciudad en toda su dimensión que tienen como un derecho los ciudadanos, pone énfasis en principios de sustentabilidad y equilibrio en las función social y ambiental, de igual manera, el artículo 32, como derecho de los ciudadanos a la salud y lo que ello implica, tal es el caso del derecho al agua que se correlaciona con el artículo 12, el derecho a la alimentación que se correlaciona con el artículo 15, el derecho a los ambientes sanos que se correlaciona con el artículo 14. Es preciso resaltar que la constitución de Montecristi, está diseñada para el Buen Vivir de los ecuatorianos en armonía con la naturaleza y el universo, a los que ha dado en llamar el paradigma del Sumak Kawsay del Ecuador.

Siendo más profundos en el análisis, encontramos para poder posteriormente trabajar la salud ambiental, el capítulo séptimo de los derechos de la naturaleza, principalmente en su artículo 71, el mismo que reza: 
"La naturaleza o Pacha Mama, donde se reproduce y realiza la vida, tiene derecho a que se respete integralmente su existencia y el mantenimiento y regeneración de sus ciclos vitales, estructura, funciones y procesos evolutivos. Toda persona, comunidad, pueblo o nacionalidad podrá exigir a la autoridad pública el cumplimiento de los derechos de la naturaleza. Para aplicar e interpretar estos derechos se observarán los principios establecidos en la Constitución, en lo que proceda. El Estado incentivará a las personas naturales y jurídicas, y a los colectivos, para que protejan la naturaleza, y promoverá el respeto a todos los elementos que forman un ecosistema". (8. p, 52).

Este artículo no permite dilaciones en cuanto al tratamiento respetuoso que merece natura de parte de los seres humanos que habitamos el Ecuador, aquí se exige el respeto máximo al planeta, conminando a todos los ciudadanos a ser actores activos en la vigilancia de los derechos de la naturaleza y otorgándoles el título simbólico de fiscales que puedan denunciar cualquier atropello que se cometa en este sentido, siendo la constitución misma la carta de garantía para que se pueda esperar el compromiso de proteger, respetar, cumplir y hacer cumplir las leyes establecidas para el efecto.

Los artículos 72, 73 y 74 son especificaciones que permiten preservar la naturaleza y a las personas del impacto ambiental que pueda sufrir el país o alguna zona en particular producto de la explotación de los recursos naturales, así también, se preservarán el ecosistema en su flora y fauna, con sus especies autóctonas y, se otorga el derecho a los ciudadanos a beneficiarse de las riquezas naturales que les permita el buen vivir.

Una vez analizados los diferentes enunciados de la carta magna ecuatoriana, se puede empezar a hilvanar desde el estudio de las políticas de salud ambiental del ministerio de salud y su dirección nacional de ambiente y salud cuya misión es "Formular y coordinar la implementación de políticas, planes, programas y demás herramientas que permitan posicionar la salud ambiental para la promoción y protección de derechos de las personas, familias y comunidades, y la generación de ambientes sanos". (2. P, 2).

Las políticas ambientales en Ecuador y la región, son específicas para cada país, sin embargo, al realizar las lecturas y análisis de las mismas se puede evidenciar que existen algunas semejanzas, por este motivo se ha elaborado un cuadro comparativo de sus objetivos: 
Cuadro 1. Objetivos de la salud ambiental en algunos países de Latinoamérica

\begin{tabular}{|c|c|}
\hline País & Objetivo \\
\hline Argentina & $\begin{array}{l}\text { Asegurar la preservación, conservación, recuperación y mejoramiento de la calidad } \\
\text { de los recursos ambientales, tanto naturales como culturales, en la realización de las } \\
\text { diferentes actividades antrópicas; Promover el mejoramiento de la calidad de vida de } \\
\text { las generaciones presentes y futuras, en forma prioritaria; }\end{array}$ \\
\hline Bolivia & $\begin{array}{l}\text { Definir acciones gubernamentales que garanticen la preservación, conservación, } \\
\text { mejoramiento y restauración de la calidad ambiental urbana y rural mediante la } \\
\text { promoción de un desarrollo sostenible con equidad y justicia social tomando en } \\
\text { cuenta la diversidad cultural del país, la conservación de la diversidad biológica } \\
\text { garantizando el mantenimiento y la permanencia de los diversos ecosistemas del } \\
\text { país optimizando y racionalización el uso de aguas, aire suelos y otros recursos } \\
\text { naturales renovables garantizando su disponibilidad a largo plazo e incorporando la } \\
\text { dimensión ambiental en los procesos del desarrollo nacional }\end{array}$ \\
\hline Perú & $\begin{array}{c}\text { Mejorar la calidad de vida de las personas, garantizando la existencia de } \\
\text { ecosistemas saludables, viables y funcionales en el largo plazo; y el desarrollo } \\
\text { sostenible del país, mediante la prevención, protección y recuperación del ambiente } \\
\text { y sus componentes, la conservación y el aprovechamiento sostenible de los recursos } \\
\text { naturales, de una manera responsable y congruente con el respeto de los derechos } \\
\text { fundamentales de la persona. }\end{array}$ \\
\hline Ecuador & Reducir la vulnerabilidad ambiental, social y económica frente al cambio climático \\
\hline Colombia & $\begin{array}{c}\text { Orientar y regular el ordenamiento ambiental del territorio y de definir las políticas y } \\
\text { regulaciones a las que se sujetarán la recuperación, conservación, protección, } \\
\text { ordenamiento, manejo, uso y aprovechamiento sostenible de los recursos naturales } \\
\text { renovables y del ambiente de la nación, a fin de asegurar el desarrollo sostenible, sin } \\
\text { perjuicio de las funciones asignadas a otros sectores }\end{array}$ \\
\hline
\end{tabular}

Fuente. Ministerios del ambiente de los países mencionados

\section{Marco conceptual}

La OMS promulgo la salud "es un estado de completo bienestar físico, mental y social, y no solamente la ausencia de afecciones o enfermedades", es una definición casi tan antigua como la organización que la promulgó, sin embargo, la esencia misma de esta definición, es clara y no permite dudas, salvo las que tenemos aquellos que sabemos lo que es salud, tan solo cuando la perdemos. La salud, es sinónimo de vitalidad y a humilde criterio se considera que para que una 
persona tenga una salud completa, esta debería estar libre de miles de microorganismos que minan a diario la salud, debería estar libre de preocupaciones que alteren su salud emocional, la tristeza, el desamor, son frecuentemente signos de una afectación invisible que afecta a la salud. La salud tiene un horizonte multidimensional imposible de visualizar, tiene que ver con factores geográficos, climáticos, de trabajo, de ingresos económicos, alimentación, educación, vivienda, de valores axiológicos y por supuesto, de la adquisición de competencias que le permiten al sujeto estar libre de pulsiones que generen el deterioro de la salud. Aspectos como los físicos, químicos, físiológicos, psicológicos, sociales y más, pueden condicionar al ser humano a estar subordinado a la salud emocional, psicológica, física o química. Hasta el factor político influye en el estado de salud del individuo. La salud no puede separarse de una serie de elementos ambientales, como el aire, agua, hacinamiento urbano, productos químicos, vectores de enfermedades, exceso de consumo, etc. Ni tampoco de las vicisitudes sociales. La creación de un ambiente adecuado o favorable para la salud depende de todos; no sólo en términos de acciones contributivas de todos los miembros, sino de acciones cooperativas inter e intrasectoriales e incluso transsectoriales $9-10$

La calidad de vida es una dimensión que es preciso analizar para posteriormente centrarnos en lo que es la salud ambiental. La calidad de vida es preciso analizarla desde las condiciones de vida del sujeto, es decir cuáles son los factores políticos, económicos, sanitarios, culturales, climáticos, etc., en donde se desenvuelve el sujeto, es decir, en qué contexto habita y evoluciona, una vez que se determine estos antecedentes, si podemos indicar, tomando la definición de la OMS que la calidad de vida desde la subjetivación del objeto de estudio, es la presencia o ausencia de satisfacción vital y desde la objetivación de parte del sujeto, es la presencia o ausencia de indicadores estándar y objetivos a satisfacer de las personas lo cual determina subjetivamente en ellos un buen vivir en un tiempo y espacio determinados.

La salud ambiental según el criterio de la Organización Mundial de la Salud, está relacionada con los factores físicos, químicos y biológicos externos de una persona, engloba factores ambientales que podrían incidir en la salud y se basa en la prevención de las enfermedades y en la creación de ambientes propicios para la salud12. La salud ambiental tiene una concepción polisémica, la misma ha ido evolucionando conforme han ido cambiando los contextos políticos, económicos y geográficos, para los años setenta del siglo pasado, fecha que coincide con nuestro análisis de las 
políticas ambientales desde las constituciones que ha tenido el Ecuador, el concepto de salud ambiental que se tenía, estaba limitado al control de los procesos químicos, físicos y biológicos, influencias o factores que ejercían efecto directo o indirecto significativo en la persona y la sociedad. Sin embargo, al ser ambigua esta definición, el órgano rector de la salud mundial, determinó que aquellos factores ambientales que ejercían influencia en la salud de las personas serían tratados desde una arista sustantiva, mientras que, la evaluación y control de los agentes que ejercían influencia en la salud de las personas por su uso y manejo, se trataría desde otra arista 13.

Garza Almanza V14, considerada uno de los teóricos sobre salud ambiental puntea que salud ambiental es: Cito (...)

"Aquella parte de las ciencias ambientales que se ocupa de los riesgos y efectos que, para la salud humana, representan el medio que habita y donde trabaja, los cambios naturales o artificiales que ese lugar manifiesta y la contaminación producida por el mismo hombre a ese medio".

Es evidente la connotación que tiene esta definición en los que analizamos la salud ambiental desde una visión humanista planetaria, pues la definición encierra al ser humano como el causante del deterioro de la salud del ambiente, ¿es el ser humano el causante de una mala salud ambiental? ¿El hombre está destruyendo la salud del ambiente donde habita? Son dos interrogantes que como especialista en salud me ronda los sueños y termino con una tercera interrogante ¿podremos plantear una alternativa que coadyuve a la mejora de la salud ambiental en la ciudad de Guayaquil, lugar de nuestra actividad profesional y personal? A pesar de ser esta definición muy precisa, considero que al decir que la salud ambiental se ocupa de los riesgos y efectos que el medio ambiente presenta para la salud humana, estamos realizando la acción de forma equivocada, deberíamos plantear los riesgos y efectos de la intervención humana en el medio ambiente que deteriora su salud (del ambiente) con la consecuencia directa del deterioro de la salud humana.

Alberto Acosta15 hace referencia que el ser humano debe aprender a convivir con la naturaleza, la misma que es generosa con nosotros, ya que nos brinda gratuitamente, una cadena alimenticia que nos permite sobrevivir, el hombre no construye su historia, es la historia la que construye la narrativa del ser humano y es este, el que debe reinscribir su actividad ecológica para poder ser 
parte del cambio que propone el estado ecuatoriano a través de la leyes de la república, señala además, que la naturaleza es dueña de sí, y que los seres humanos tenemos prestada a la naturaleza, por tanto culmina, se debe cambiar la acciones para con ella .

Rengifo Hugo16, propone que: Cito (...)

"La salud ambiental se ocupa de las interrelaciones interactivas positivas y negativas del hombre con el medio ambiente donde se habita y trabaja, incluyendo los otros seres vivos como animales y plantas, los cambios naturales o artificiales que ese lugar manifiesta y la contaminación producida por el mismo hombre en el ambiente y que puedan afectar a la salud humana, así como su estrecha relación con el desarrollo sostenible". (16.p, 4).

\section{Campos de atención de la salud ambiental.}

1. Contaminación ambiental. Dentro de este amplio campo consideramos la contaminación del aire que incluye tanto el aire externo como el que está dentro de las viviendas o aire interior; toda forma de radiaciones como el ruido, la lumínica y las radiaciones ionizantes y no ionizantes como los campos electromagnéticos, la contaminación del agua y la contaminación del suelo.

2. Saneamiento ambiental. Comprende la provisión de agua potable y alcantarillado además del manejo y disposición de residuos y excretas tanto sólidas y líquidas.

3. Enfermedades y vectores. Incluye las enfermedades emergentes y reemergentes, además del control de vectores y zoonosis.

4. Nutrición. Incluye la seguridad alimentaria en la cadena alimentaria, como los aspectos nutricionales, con especial énfasis en la recuperación de hábitos alimenticios ancestrales y uso de alimentos nativos, reemplazando a los foráneos similares.

5. Sustancias químicas tóxicas. Manejo y control adecuados y su toxicología.

6. Hábitat saludable. Dentro de este campo se estudia el síndrome del edificio enfermo, el hacinamiento y tugurización, las escuelas, hospitales y ciudades saludables, la contaminación paisajista o eyesore, la agresión al ciudadano -servicios públicos, obras viales privadas y públicas-, arquitectura agresiva al entorno, inaccesibilidad a locales de servicios, la depredación 
y deforestación de áreas verdes urbanas y rurales y la fitoremediación de gran importancia en la descontaminación.

7. Vulnerabilidades sociales. En esta área se analiza la violencia, la pobreza extrema y pobreza límite, las migraciones internas, el trabajo saludable (con el área de la salud ocupacional), transporte adecuado y saludable.

\section{Desastres. Antropogénicos, su previsión y manejo.}

9. Multiculturalidad. Se estudia los acervos tradicionales de y en salud ambiental, sistematizando los conocimientos populares y comprendiendo las diferentes cosmovisiones para la solución de los conflictos ambientales que involucren problemas de salud ambiental, además del estudio de recursos biológicos (flora y fauna) y naturales de uso ancestral.

10. Educación para la salud ambiental. En todos los niveles.

11. Nuevas biotecnologías. Como la nanotecnología, los organismos genéticamente modificados, la biología sintética y la ingeniería genética cuyos rápidos avances y aplicaciones, muchas veces dejan de lado el principio de precaución.

\section{Biodiversidad. Y protección a los ecosistemas.}

13. Gestión de la salud ambiental. Comprendiendo tanto el diseño de políticas, planes, estrategias, como una vigilancia y control adecuados, teniendo presente los principios de la ética de la salud ambiental y el respeto a los derechos universales.

La ciudad de Guayaquil, tiene características muy particulares: encontrase en la región costa, a la cuenca del rio Guayas, ser el eje de desarrollo económico del país, ser la ciudad de mayor captación de migración rural del Ecuador, entre otras constituyen factores a tomar en cuenta a la hora de señalar el diagnostico de salud ambiental de la urbe porteña. El mayor asentamiento humano del Ecuador se encuentra en la ciudad de Guayaquil, es el mayor centro de migración del país, por este motivo, se presenta un desorden urbanístico, deficiente prestación sanitaria, insuficiente prestación de servicios de agua potable y alcantarillado. Dentro de este contexto, la situación de la salud ambiental en la ciudad pasa por la misión que tiene el municipio de la urbe en este sentido "Promover y asegurar la gestión ambiental integral dentro del ámbito competente, priorizando la conservación de los recursos naturales del cantón, el desarrollo de una conciencia 
ambiental ciudadana y manteniendo el espíritu de servicio”, además tiene el encargo de planificar, dirigir, coordinar y supervisar la implementación de proyectos de desarrollo sostenible dirigidos a la protección de las áreas naturales del cantón Guayaquil y la restauración de ecosistemas vulnerables y; sus líneas de acción son: Conservación y recuperación de áreas naturales de importancia ecosistémica; Proyectos de desarrollo sostenible; Sensibilización y educación ambiental.

Las políticas de salud ambiental del municipio de Guayaquil, incorpora a su plan de trabajo el departamento de medio ambiente y con el genera una políticas en donde el desarrollo humano sostenible se convierte en el eje transversal del municipio, para ello genera programas y proyectos, en donde se procura que sus habitantes mantengan una convivencia armónica con el medio natural, así también, búscala participación ciudadana en cuanto al mejoramiento de la calidad ambiental y la restauración de los ecosistemas degradados, con esto, se pretende lograr un aprovechamiento sustentable de sus recursos naturales, manteniendo los procesos ecológicos propios y compartiendo su experiencia en gestión ambiental con los cantones vecinos.

Es saludable reconocer que las políticas del municipio de Guayaquil, en el artículo 4 se encuentran las siguientes:

- $\quad$ Promover y colaborar en la formulación -y ejecución en función de sus posibilidades y en el ámbito de su jurisdicción- de un Plan de Manejo de la bioregión del Golfo de Guayaquil, en cuyo marco sea posible maximizar los beneficios ambientales que reportan el río Guayas y el Estero Salado, procurando la recuperación de las áreas degradadas, gestionando racionalmente sus capacidades productivas y ambientales y procurando la sustentabilidad de los sectores económicos que dependen de las mismas.

- Procurar el uso sostenible de los recursos hídricos del cantón, por ser beneficios ambientales esenciales y constituirse en uno de los ejes de la calidad de vida de la ciudad.

- Integrar sustentablemente la acuacultura y las pesquerías con el sistema estuario del Río Guayas de tal forma que los recursos bioacuáticos, importantes activos del cantón, sean explotados racional, técnica y sosteniblemente, constituyéndose en uno de los principales motores de la conservación y mejora de los manglares del Golfo de Guayaquil. 
- Controlar y disminuir la tasa de deforestación de los bosques nativos del cantón y de las áreas naturales y coordinar acciones tendientes a forestar y reforestar las zonas afectadas.

- Adoptar un esquema responsable de conservación estricta de ecosistemas frágiles como manglares, humedales y bosques secos tropicales, así como también implementar acciones de restauración de poblaciones de especies de fauna y flora endémicas y nativas de los ecosistemas mencionados.

- Crear un entorno de conciencia ambiental adecuado en los habitantes del cantón y en los sectores productivos, de tal forma que asuman responsabilidades ambientales y arbitren sistemas de autocontrol, implantando procesos de producción más limpia y dispositivos de reducción de emisiones y sistemas de depuración en las industrias.

- Planificar el desarrollo de la ciudad respetando sus recursos naturales y facilitando el desarrollo de la vida cotidiana de sus habitantes y de sus visitantes.

- Desarrollar en el cantón Guayaquil un sistema de parques naturales y áreas verdes capaces de responder a las necesidades de recreación, y mantener los hábitats naturales y la vida silvestre.

- $\quad$ Fomentar el uso de energía renovable en la ciudad.

- $\quad$ El buen manejo de los recursos hídricos y la prevención y control de la contaminación de los mismos, así como del capital natural del cantón, de conformidad con la normativa jurídica pertinente.

- $\quad$ Procurar el desarrollo de una identidad ambiental comunitaria fuerte.

- Coordinar mecanismos de cooperación ambiental con los cantones limítrofes, las instituciones pertinentes de ámbito provincial, regional y nacional, respetando la autonomía de cada institución y aprovechando los potenciales y vocaciones locales.

Las políticas de salud ambiental en el municipio de Guayaquil, son, al igual que la constitución ecuatoriana, retórica muerta en el papel, esto se argumenta desde la observación empírica que durante más de cuarenta años se realiza en la ciudad, en donde el abandono de los anillos periféricos continúan gobierno tras gobierno, en donde no se presta atención a los servicios básicos de los asentamientos humanos de la urbe, en donde se realiza una regeneración urbana de 
la zona comercial céntrica de la ciudad. Desde la praxis profesional, se emite un juicio subjetivo de la realidad ambiental de la ciudad, esto debido a los más de 30 años de docente en la Universidad de Guayaquil, lo cual ha permitido tener contacto con la comunidad, a través de la vinculación con la comunidad como uno de los ejes sustantivos de la Universidad de Guayaquil y que esta como parte del rol docente.

\section{Discusión}

La salud ambiental es una deuda pendiente en el Ecuador, dentro del marco normativo, el gobierno nacional a través de la secretaria de salud y secretaria del medo ambiente, no priorizan la salud ambiental, sino que, estas son implementadas como resultantes de los efectos que un fenómeno determinado ocasiona, es decir tan solo se implementan políticas y programas para apagar incendios. Existe una escasa determinación de parte del gobierno por otorgar los recursos necesarios al sector salud humana y salud ambiental, se resta importancia a los lineamientos constitucionales del Sumak Kawsay y se irrespeta a la Pacha Mama, además, se limitan los compromisos adquiridos en el campo de la política internacional en cuanto al cuidado de la salud ambiental. Se concierta con los países de la región cuyos objetivos generales son, al igual que los objetivos del Ecuador y la ciudad de Guayaquil, el preservar la salud humana desde la salud ambiental. En cuanto se refiere al país, desde la década de los 70 en el siglo pasado hasta la fecha, tan solo se han presentado leyes en papel de como preservar la salud ambiental de la patria, son narrativas con tinte demagógico que si bien es cierto están institucionalizadas en la constitución, no se ha construido una arquitectura teórico-práctica que dé respuesta a la demanda social de Montecristi. En los años 2008-2013, se violó de forma constante la nueva constitución en cuanto al respeto de los artículos 12,13, 14 y 15 de buen vivir de los ecuatorianos; se vulneró el artículo 31 ya que no se pudo disfrutar de un ambiente saludable en las ciudades y en especial en las zonas de explotación minera y petrolera, se desalojó a pueblos indígenas enteros para otorgarle a transnacionales chinas la explotación indiscriminada y sin presentar programas de explotación sin impacto ambiental de los recursos naturales, produciendo con esto, daño a la función social que reza en la constitución. De igual forma con este hecho, se dislocó la esencia del artículo 32 sobre la salud de las poblaciones que habitaban en las zonas de explotación petrolera y minera, ya que, los daños en la salud son a largo plazo20. 
En cuanto al artículo 71, en el gobierno anterior y de turno, no se respetó la Pacha Mama tal como rezaba que se haría en la constitución de Montecristi, con el atenuante que no se podía reclamar los derechos de la naturaleza porque quien lo hacía, era considerado traidor a la patria, quedando la naturaleza desprotegida en cuanto a su salud ambiental y con ella la población.

La ciudad de Guayaquil presenta propuestas para una salud ambiental que distan de la realidad que se vive en la urbe porteña, aún se espera que desaparezcan los 25 focos críticos de contaminación existentes en la ciudad 21. Así de igual forma aún persiste la deuda en la provincia del Guayas con el ambiente en cuanto al tratamiento de aguas 20. También, se sigue presentando como una ducha de pesticidas, muchos de ellos prohibidos en la UE y Dinamarca, debido a que se sigue rociando los pesticidas desde avionetas en las plantaciones de banano, arroz y cacao, son un total de 26 pesticidas que se rocía en las plantaciones en las plantaciones agrícolas de la costa ecuatoriana 22 .

Para concluir las políticas que se han presentado desde la década de los setenta en el siglo pasado, han sido demagógicas, ninguna de ellas se ha cumplido porque ha primado el principio de desarrollo económico, en detrimento del desarrollo humano y planetario. No existen en la actualidad programas serios que permitan tener fe en que se devuelva la salud ambiental perdida en el país. Así de igual forma, en la ciudad de Guayaquil no se respeta la biodiversidad, los ecosistemas son vulnerados y se transgrede la naturaleza.

En cuanto al Sumak Kawsay, en los artículos de la constitución que velan por mantener el romance entre Pacha Mama y el ser humano, son letra que reposan sin llegar a despertar para convertirse en una práctica social responsable y solidaria con el planeta y con nosotros mismos, la forma de hacerlo está en lo que se argumentó al principio, en lograr decolonizarnos como docentes universitarios para de esta forma poder desarraigar la colonialidad existente en el constructo cognitivos de la masa social ecuatoriana, para ello, es preciso que nos empoderemos del objeto del conocimiento con una epistemología desde el sur en cuanto al objeto de estudio 23.

\section{Propuesta}

Descolonizar la docencia universitaria y desde allí y a través de la pedagogía, instaurar una nueva cosmovisión humanista y planetaria que permita romper la colonialidad existente en el constructo socio-cognitivo de la sociedad, siendo la juventud que ingresa a las aulas universitarias, el agente 
a cambiar y nosotros el leudante de ese cambio. Si se alcanza la decolonización, se habrá dado el primer paso, el segundo paso es hacer de esa generación que tomemos para enseñar desde el Sursea una generación libre, invito a este diálogo al maestro de la película el lenguaje de las mariposas quien dice "si conseguimos que una generación, una sola generación, crezca libre en España, ya nadie les podrá arrancar nunca la libertad...Nadie les podrá robar ese tesoro”, lo extrapolo al contexto latinoamericano, y pienso en voz alta y escribo: si conseguimos que una generación, una sola generación, crezca con libertad cognitiva en Latinoamérica, ya nadie les podrá arrancar nunca la libertad. Nadie les podrá robar ese tesoro

Preparación con responsabilidad y bajo sólidos principios axiológicos, con el empoderamiento metacognitivos del objeto de conocimiento que permita construir desde la episteme del objeto.

Esta propuesta atrevida e irreverente, queda como una chispa, para que desde ella, se generen propuestas más acercadas al objeto de estudio de la presente investigación.

\section{Referencias Bibliográficas}

Roa A L, Pescador Vargas B. La salud del ser humano y su armonía con el ambiente2016. [Citado 2018 Dic 19]. Disponible en: https://dialnet.unirioja.es/servlet/articulo?codigo=5676827

Ramírez R. Transformar la universidad para transformar la sociedad (segunda ed.). Quito: Secretaría Nacional de Educación Superior. 2012. ISBN: 978-9942-07-243-6

Ojeda del Valle Mayra. Salud, ambiente y desarrollo: a propósito de una investigación de salud escolar. Rev Cubana Hig Epidemiol. 2012 [citado 2018 Dic 19]; 50 (3): 392-402. Disponible en: http://scielo.sld.cu/scielo.php?script=sci_arttext\&pid=S1561-30032012000300013\&lng=es.

GEOECUADOR. Políticas ambientales. 2008 [citado 2018 Dic 19]. Disponible en: https://goo.gl/ptzxHr

Ecuador. Constitución ecuatoriana de 1968 [citado 2018 Dic 19]. Asamblea 25 de mayo de 1967. Disponible en: https://goo.gl/d3LfxN

Ecuador. CONSTITUCION POLITICA DEL AÑO 1979. Decreto Supremo 000, Registro Oficial 800 de 27 de marzo de 1979 [citado 2018 Dic 19]. Disponible en: https://goo.gl/2Wp2Bz. 
Landázuri E, Jijón C. El Medio Ambiente en el Ecuador. Quito: ILDIS; 1998 [citado 2018 Dic 19]. Disponible en: https://goo.gl/DSKtSz

Cueva, A. Ecuador en su nueva encrucijada histórica. Cuadernos Políticos, (27), 49-54. 1979.

Organización Mundial de la Salud. Constitución de la Organización Mundial de la Salud. Nueva York: OMS; 1946 [citado 2018 Dic 19]. Disponible en: https://goo.gl/oc4WDa

Organización Mundial de la Salud. Salud, medio ambiente y desarrollo; enfoques para la preparación de estrategias a nivel de países para el bienestar humano, según la Agenda 21. Ginebra: OMS; 1994.

Urzúa A, Caqueo-Urízar A. Quality of life: A theoretical review. Escuela de Psicología, Universidad Católica del Norte, Chile. Depto. de Filosofía y Psicología, Universidad de Tarapacá, Chile. 2012 [citado 2018 Dic 19] ; 61-71. Disponible en: https://goo.gl/6rxUT9

OMS. Salud ambiental - World Health Organization. 2018. [citado 2018 Dic 19] Disponible en: https://goo.gl/ZtLsgm

Ordoñez GA. Salud ambiental: conceptos y actividades. Rev Panam Salud Pública. 2000; 7 (3): $137-47$

Garza-Almanza V. Salud y ambiente en el desarrollo sostenible. Ambiente sin Fronteras.1997; $1(6): 1-6$.

Acosta A. La naturaleza como sujeta de derechos [documento en internet]. Quito: Asamblea Constituyente; 2008 [citado 2018 Dic 19] Disponible en: http://asambleaconstituyente.gov.ec/blogs/alberto_acosta/2008/02/29/la-naturaleza-comosujetade-derechos/

Rengifo Cuéllar Hugo. Conceptualización de la salud ambiental: teoría y práctica (parte 1). Rev. Perú. Med. Exp. Salud pública. 2008 [citado 2018 Dic 19]; 25 (4): 403-409. Disponible en: http://www.scielo.org.pe/scielo.php?script=sci_arttext\&pid=S1726$46342008000400010 \& \operatorname{lng}=$ es.

República del Ecuador.2000 [citado 2018 Dic 19]. Disponible en. https://www.oas.org/dsd/publications/Unit/oea60s/oea60s.pdf 
Municipalidad de Guayaquil. Políticas ambientales.2018 [citado 2018 Dic 19]. Disponible en: https://www.guayaquil.gob.ec/pol\%C3\%ADticas-ambientales

Aguilar D. Desalojo a una comunidad shuar de la Amazonía aviva resistencia indígena contra la gran minería. Mongabay Latam, Periodismo ambiental independiente, 2016 [citado 2018 Dic 19]. Disponible en: https://goo.gl/d5Mx75

El Universo. Guayaquil tiene más de 25 puntos críticos de contaminación 2005 [citado 2018 Dic 19]. Disponible en: https://goo.gl/AAiAcS

Avilés M. Guayas: el tratamiento de aguas, en deuda con el ambiente. EXPRESO. 2018 [citado 2018 Dic 19]. Disponible en:https://www.expreso.ec/actualidad/guayas-aguas-residualestratamiento-salud-medioambiente-KJ2290048

Osefsen L, Calabria R. vivir y morir del banano. PLAN V. ECUADOR, 2018 [citado 2018 Dic 19]. Disponible en: http://www.planv.com.ec/investigacion/investigacion/vivir-y-morir-delbanano.

Vargas T, Mendieta L. Decolonizar la docencia Universitaria Ecuatoriana. Polo del Conocimiento, DOI: https://doi.org/10.23857/pocaip. Disponible en: https://goo.gl/qUNXoP

En el año 2013, la película el lenguaje de las mariposas fue interpretado por Fernando Fernán Gómez y en su discurso de despedida dijo "Dignísimas autoridades, queridos niños, respetados convecinos. En la primavera el ánade salvaje vuelve a su tierra para las nupcias. Nada ni nadie podrá detenerlo: Si le cortan las alas irá a nado; si le cortan las patas se impulsará con el pico, como un remo en la corriente. Ese viaje es su razón de ser. En el otoño de mi vida, yo debería ser un escéptico y en cierto modo... Lo soy. El lobo nunca dormirá en la misma cama con el cordero; pero de algo estoy seguro, si conseguimos que una generación, una sola generación, crezca libre en España, ya nadie les podrá arrancar nunca la libertad. Nadie les podrá robar ese tesoro". Disponible en: http://educomunicacion.es/cineyeducacion/temasmariposas.htm 